\title{
Las barreras para la entrada de competidores potenciales a los sectores de actividad y su influencia en la posibilidad de obtener beneficios en los mismos Barriers to new entry and their influence over the potencial benefits in the industry \\ Gustavo Mata Fernández-Balbuena \\ Escuela de Negocios IEDE. Universidad Europea de Madrid. C/ del Tajo. El Bosque, Villaviciosa de Odón. 28670 Madrid.
}

Fecha de recepción: 20-04-08

Fecha de aceptación: 10-07-08

Resumen. Pese a la trascendencia que tiene en el análisis de los sectores de actividad y su competencia de Porter, el concepto de barreras a la entrada de nuevos competidores, no es fácil de precisar, ni tampoco es fácil definir qué es lo que se entiende por tamaño de las barreras. Tampoco la posibilidad para los instalados de lograr beneficios en un sector está biunívocamente relacionada con la existencia de tales barreras, ni la existencia de las barreras se puede subsumir en la existencia de un precio disuasorio para los competidores potenciales que, sin embargo, permitiera a los instalados tener beneficios, como sostiene también el Profesor Porter. Las causas y los efectos de cada tipo de barrera son muy diversas; para analizar correctamente su papel será necesario estudiar cada causa de forma individual y, después, estudiar los posibles efectos agregados de las mismas. El análisis de cómo las barreras al acceso pueden influir en la posibilidad de que haya beneficios en el sector de actividad no se puede resolver con una síntesis integrativa simplificadora.

Palabras clave: barreras de entrada, precios disuasorios al ingreso, posibilidad de tener beneficios.

\begin{abstract}
In spite of the importance that the analysis of the industries and competitors from M.E. Porter has, the concept of barriers is not easy to define, neither is it easy to define the size of the barriers. The entry barriers do not exactly determine the possibilities of making profits, and the existence of the barriers do not necessarily mean a dissuasive price for the potential competitors, however they do allow the installed ones to have benefits, as Professor Porter says. The causes and the effects of each type of barrier are very diverse; in order to correctly analyze their role it will be necessary to study each one individually, and later to study the possible added effects. The analysis of the relationship between barriers to the new entry and benefits in the industry cannot be solved with a simplifying integrative synthesis.
\end{abstract}

Key words: barriers to new entry, dissuasive low prices to the entrance, benefits.

\section{El concepto de barrera de entrada}

M. E. Porter' define las barreras de entrada a un sector como todo aquello que impide o dificulta el acceso de los competidores potenciales al mismo y cita como posibles causas de las barreras: las economías de escala, los costes conjuntos aplicables a más de una actividad, la diferenciación de los productos, las necesidades de capital para instalarse, los costos que habrían de asumir los clientes por cambiar de proveedor, el acceso restringido a los canales de dis- tribución, las desventajas en coste independientes de las economías de escala - ubicación favorable, acceso preferencial a primeras materias, tecnología de productos patentada, subsidios gubernamentales, curva de aprendizaje o de experiencia- y las políticas de la administración.

Para Porter, la posibilidad de obtener beneficios en un sector está relacionada con las barreras de acceso al mismo. También para él, la «condición de entrada» a un sector industrial puede resumirse en un

\footnotetext{
I Porter, M.E. (1982). Competitive Strategy. Techniqes for Analyzing Industries and Competitors. New York, The Fress Press
} 
concepto: el «precio disuasorio» al ingreso; si los instalados mantuviesen los precios por debajo de ese hipotético precio disuasorio se podría eliminar la posibilidad de nuevos entrantes.

El trabajo pionero sobre barreras de entrada se debe a J. S. Bain². Bastantes años antes de que lo hiciera Porter, Bain había definido las barreras de acceso como aquellas condiciones que permiten que los instalados en el sector puedan elevar los precios por encima de sus costes - es decir que puedan tener beneficios - sin por ello poner el ingreso al alcance de nuevos entrantes o competidores potenciales. Bain define el concepto por sus consecuencias; está claro que entre barreras de entrada, en el sentido de Bain, y posibilidad de tener beneficios hay una correlación total.

Para Bain el tamaño de las barreras está relacionado con el «precio máximo» —aquel que podrían los instalados practicar sin poner por ello la entrada al alcance de sus competidores potenciales_-; para él la «condición de entrada» - el margen porcentual del precio máximo sobre el coste medio mínimo alcanzable por las empresas instaladas - sería lo que mediría el tamaño de la barrera.

Las definiciones de barreras de Porter y de Bain no son idénticas ni siquiera equivalentes; no es lo mismo un impedimento u obstáculo a la entrada, que una condición que permita que los instalados en el sector tengan beneficios, sin poner por ello el ingreso al alcance de los competidores potenciales. Si las barreras fueran lo que define Bain, como las barreras son la condición de que haya posibilidad de beneficios, no sólo estarían relacionadas: serían lo mismo. Si fueran lo que define Porter como tales, no sería exactamente cierto que la posibilidad de tener beneficios estuviera relacionada con su existencia como él mantiene. Trataremos de explicarlo.

\section{Las causas de las barreras de entrada}

Las causas para las barreras que señala Porter se pueden agrupar, conceptualmente, en nuestra opinión, en cinco grupos:

- Las que se derivan de la exclusividad: exclusivo es el que excluye, el único que tiene una característica; un producto sin sucedáneos ni sustitutivos posibles, o un acceso exclusivo a la única distribución posible.
- Las que se derivan de la necesidad de una fuerte inversión: o mejor, de la existencia de grandes costes sumergidos, naturales o forzados, pero no recuperables, irreversibles, para acceder al sector.

- Las derivadas de ventajas en coste para los instalados independientemente de las economías de escala: siempre que estén fuera del alcance de los competidores potenciales, como procesos ventajosos para el coste protegidos por patentes, ventajas derivadas del efecto de la curva de experiencia, acceso a menores costes por tenerlos compartidos con otras producciones, acceso a primeras materias en condiciones ventajosas o a las únicas ubicaciones favorables, etc.

- Las derivadas de las economías de escala: que operarían como o tales barreras sólo cuando la escala eficiente fuera del orden del tamaño del mercado y sólo cupieran un pequeño número de concurrentes en él, siempre, además, que actuasen de hecho como un cártel al reconocer su mutua dependencia.

- Las que provoca la diferenciación de productos en el sector: cuando todos los productos fueran parecidos, pero, para cada segmento, unos fueran preferibles a los otros que serían sustitutivos más o menos próximos pero no perfectos del preferido, lo que puede ocurrir tanto por cuestiones de fondo como de forma - en el caso de que la diferenciación procediera de una identificación formal lograda a través de la creación de marcas--. Aquí se podrían incluir también las barreras originadas por el coste que tuviera para el cliente cambiar de proveedor que son, esencialmente, como las barreras por diferenciación - también al que se acostumbra a un producto, le «cuesta» cambiar a un sustitutivo próximo-. Para que esta diferenciación operara como barrera tendría que ir asociada a una relativa estanqueidad de cada segmento y a economías de escala que, aunque no fueran válidas como barrera para el mercado global sí pudieran ser relevantes en un segmento diferenciado y relativamente estanco, como tales

\section{La naturaleza y los efectos de las barreras}

La naturaleza y los efectos de los distintos tipos de barrera señalados es muy diversa y su análisis no es

\footnotetext{
2 Bain, J.S. (1968). Barriers to new competiton. Cambridge Mass, Harvard University Press
} 
sencillo. Unas barreras son absolutas pues impiden totalmente el acceso —las derivadas de la exclusividad-y otras relativas, pues disuadirían pero no impedirían «strictu sensu» el acceso —como los grandes costes hundidos, las ventajas en coste, las economías de escala o la diferenciación de los productos en el sector.

De otra parte, unas barreras están basadas en que la entrada esté restringida o impedida por algún obstáculo identificable - imposibilidad de fabricar el producto, imposibilidad de alcanzar los mismos costes, necesidad de realizar una fuerte inversión irrecuperable para acceder - y otras se configuran de forma derivada sin que haya impedimentos, «a prioi», al acceso - en principio, todos tendrían a su alcance las mismas condiciones y la única diferencia estaría en que unos ya estarían dentro y los otros no-, como cuando hay fuertes economías de escala o cuando la diferenciación hace que en cada segmento relativamente estanco puedan actuar unas economías de escala que no serían relevantes para el mercado global y sí para cada segmento.

\section{La posibilidad de tener beneficios y las barreras}

Desde luego no se puede decir que a mayores barreras de entrada, en el sentido de Porter, habrá mayores beneficios. La existencia de barreras puede ser una condición necesaria pero no es una condición suficiente para que haya beneficios -al menos, está claro que incluso por tener un monopolio por el control absoluto de algún factor, o por la exclusividad del propio producto, no se asegura el beneficio, pues haría falta para tenerlo que, adicionalmente, la curva de demanda del producto fuera secante a la curva de costes medios del monopolista (condición de cierre para el monopolista) - si así no fuera, no habría beneficios para el instalado, y la barrera: ¡no podría ser mayor!; la entrada estaría impedida.

La existencia, natural o forzada, de grandes costes sumergidos en el sector — costes irrecuperables - actuaría frenado a posibles concurrentes, aunque no impidiendo absolutamente la entrada, como vimos más arriba. En este caso, aunque las barreras no fueran absolutas, sí resultarían en muchos casos suficientemente disuasorias por la asimetría de condiciones entre los ya instalados y los competidores potenciales. Ahora bien, en el grupo de los ya insta- lados podría haber o no beneficios, aunque la entrada de nuevos competidores esté disuadida. Podrá haber beneficios si es un pequeño grupo que reconociendo su interdependencia actuara como un cártel, si no, lo probable es que todos se enzarcen en una guerra de precios que acabe con todos ellos vendiendo sin márgenes.

De otra parte, cuando hubiera libertad de acceso y de salida al sector, cuando no hubiera obstáculos o impedimentos previos, cuando todos los competidores pudieran acceder a los factores de producción en condiciones de igualdad y la información circulase libremente, con lo que todos tendrían acceso a las mejores curvas de costes — las idóneas-, los instalados podrían contar con posibilidades de disuadir de algún modo el acceso de nuevos concurrentes. También sin obstáculos a la entrada o salida de concurrentes puede haber beneficios en el sector. Sería el caso cuando hubiera economías de escala -independientemente de que todos pudieran acceder a las mismas curvas de coste puede haberlas-; las economías de escala pueden originar barreras sólo si éstas fueran tales que dieran lugar a que en el sector hubiera sólo un grupo muy pequeño de empresas y sólo si éstas reconocieran su mutua interdependencia actuando como un cártel frente al mercado. La diferenciación tampoco crea, por sí misma, barreras de acceso, como se deduce claramente en el modelo de la competencia monopolística de E. H. Chamberlin ${ }^{3}$; sólo en el caso de que la diferenciación fuera tan extrema que se diera que en el segmento al que se dirigiera la modalidad de producto diferenciado no hubiera sustitutivos efectivos, y que, por tanto, el segmento fuera un compartimento estanco, se podría configurar en ese segmento un pequeño grupo en el que las economías de escala, que tal vez, si las hubiera, no fueran suficientes para crear barrera en el mercado global, sí se hicieran valer de forma efectiva en el compartimento estanco. La diferenciación para poder actuar como barrera necesita de las economías de escala, si no, no las llega a crear.

En el modelo de Porter no queda claro qué se entiende por tamaño de las barreras, y, como hemos visto, no habría una correlación clara entre el tamaño de las barreras de acceso a un sector y la cuantía de los beneficios obtenibles en él; relacionar el tamaño de las barreras de entrada con los beneficios esperables en el sector es, además de poco riguroso - por la falta de precisión en el concepto de tamaño de la barrera-, erróneo.

\footnotetext{
${ }^{3}$ Chamberlin, E.H. (1966). The Theory of Monopolistic Competition. Cambridge, Mass., Harvard University Press
} 


\section{Los precios disuasorios a la entrada y las barreras}

En nuestra opinión, tampoco se sostiene la afirmación de Porter y de Bain de que, finalmente, la existencia de barreras a la entrada se puede resumir en la posibilidad de practicar precios disuasorios para el ingreso.

En primer lugar, hay veces en las que no es posible el acceso de competidores, independientemente del precio que se practique en el mercado: un monopolista - o un grupo de oligopolistas - que dominara un factor clave o que fuera el único que pudiera fabricar un producto sin sustitutivos no pondría el acceso al alcance de ningún competidor potencial, practicara el precio que practicara.

En segundo lugar, en un monopolio natural, que tuviera barreras al acceso por economías de escala, el monopolista podría estar practicando el precio óptimo para él, sin por ello facilitar la entrada de competidores - en este caso, disuadiéndola aunque sin poder impedirla absolutamente: el segundo podría entrar y ambos entrarían en pérdidas, hasta que uno de los dos saliera, pero no, necesariamente, tendría por qué ser él-. En cualquier caso, aunque en el sector hubiera uno y pudiera haber beneficios para dos, con lo que no habría ya barrera por economías de escala para la posible entrada del segundo, el instalado no lograría nada bajando el precio para disuadir la entrada del competidor potencial: pues si lo hiciera, cuando el segundo entrase, ambos tendrían, necesariamente, que subirlos; haber bajado el precio habría sido ineficaz para impedir o dificultar la entrada, no habría precio disuasorio, habría sido una señal falsa o una amenaza vana.
Cuando uno o algunos de entre los instalados tuvieran mejores costes que los demás, sí tendría, o tendrían, la posibilidad de restringir la competencia en el sector bajando los precios, dificultando así, aunque no impidiendo, en sentido estricto, la entrada de competidores potenciales y procurando la salida de los que no accedieran a esos costes bajos. Pero, en estos casos de ventajas absolutas en coste, los beneficios máximos para los que tuvieran ventaja no se obtendrían impidiendo o dificultando la entrada de competidores potenciales — para lo que haría falta bajar los precios por debajo de los costes mínimos de ellos-, sino que, actuando como cártel o como empresa dominante ${ }^{4}$, éstos se conseguirían manejando el precio en el mercado - lo que se lograría deduciendo de la recta de demanda global la curva de oferta del resto de concurrentes, para calcular así la demanda a su alcance a cada precio y deducir cuál sería su curva de ingresos marginales, para buscar entonces la cantidad adecuada a ofertar para la que éstos coincidieran con su coste marginal.

\section{Conclusiones}

El que haya barreras de entrada a un sector no es exactamente lo mismo que la falta de libertad de acceso al mismo y tampoco es lo mismo que haya barreras, en el sentido de Porter, que tener la posibilidad de beneficios. Tampoco las barreras están relacionadas, directamente, con un hipotético precio disuasorio que podrían practicar los instalados sin poner por ello el ingreso al alcance de los competidores potenciales como sostienen Porter y Bain. $\mathrm{Ni}$ su análisis es riguroso ni sus conclusiones son sostenibles.

\footnotetext{
${ }^{4}$ Este modelo, referenciado por diversos autores -Clarke, R. (1993). Economía industrial. Colegio de Economistas, Celeste Ediciones, págs. 72 y 73; Pindick, R.S., y Rubinfeld, D.L. (200 I). Microeconomía (5ª ed.). Pearsons Educacion, S.A., Madrid, págs. 463 a 469- es utilizado en el análisis de los sectores de actividad dominados por un cártel formado por un número reducido de ofertantes por Saving, T. R. (1970). «Concentration ratios and the degree of monopoly». International Economic Review, I I, págs. I 39 a I 46.
} 